\title{
Parallel perceptual processing and decisional strategies: A reinterpretation of the Shaw and LaBerge effect*
}

\author{
GERALD T. GARDNER \\ Unilersity of Michigan-Dearborn, Dearborn, Michigan 48128
}

\begin{abstract}
A reinterpretation is offered for an effect found by Shaw and LaBerge (1971) in a tachistoscopic detection paradigm. Their Ss were instructed to "scan" the letters of a multiletter stimulus array in a particular sequence. The Ss were more accurate in identifying a target letter if it occurred earlier rather than later in the instructed sequence. Although Shaw and LaBerge interpreted this result as supporting a limited-capacity attentional mechanism, the result is also consistent with a nonattentional parallel processing conception: the payoff incentives used to encourage the correct "scanning sequence" could alter Ss" postperceptual decisional strategy so as to produce the results obtained. Given the compatibility of a parallel processing conception with the results of Shaw and LaBerge and the results of other detection experiments, the need for postulating a spatial capacity limit or attentional mechanism is questioned.
\end{abstract}

Recently, Eriksen and Spencer (1969), Gardner (1970, 1973), and Shiffrin and Gardner (1972) have accumulated evidence for the operation of independent parallel channels in tachistoscopic perception. These researchers conclude that the processing underlying a visual detection task (e.g., Estes \& Taylor, 1966) entails no spatial capacity limit or voluntary attentional control. A finding by Shaw and LaBerge (1971), however, appears to pose a problem for this conclusion. Shaw and LaBerge required Ss to identify a single target letter embedded in a multiletter tachistoscopic array; Ss were instructed to "read" or "scan" the letters of the array in a particular ordinal sequence. The results indicated that, with sufficient practice, target identification was more accurate when the target came earlier rather than later in the instructed sequence. Shaw and LaBerge concluded that processing of the arrays involved an attentional bottleneck-most likely a serial mechanism operating until the cessation of stimulus persistence; the earlier the location of the target in the attentional sequence, the greater the probability of its being processed successfully. The Shaw and LaBerge results, however, may be reinterpreted in a manner consistent with a parallel-channels, nonattentional conception of visual processing. The present paper at tempts to develop this reinterpretation.

Shaw and LaBerge (Experiment 2) used seven-letter stimulus arrays arranged in a triangular format: three letters appeared as a group near the point of fixation; two letters appeared as a group above and to the right of the first group: the remaining two letters appeared as a group below and to the right of the first group. The

*This work was supported by Rackham Faculty Research Grant FRR 1004 and Office of Research Administration Grant 360345 from The Lniversity of Michigan, Ann Arbor. The author thanks Howard Egeth and William Thomson for helpful comments on earlier drafts of this paper.

$\div$ Requests for reprints should be sent to Gerald T. Gardner. Department of Psychology, The University of Michigan-Dearborn. Dearborn. Michigan 48128. target letter (A, T, or $U$ ) was located in either a prespecified location of the upper two-letter group or a prespecified location of the lower two-letter group, according to a random schedule. In the clockwise condition, Ss were instructed to "scan" the array from fixation group to upper group to lower group; in the counterclockwise condition, Ss were to "scan" from fixation group to lower group to upper group. To encourage these orders, Ss were given point incentives, the $\mathrm{S}$ accumulating the greatest point total receiving a $\mathrm{S} 5$ bonus at the end of the experiment. In the clockwise condition, Ss received 4 points for correct target identification if the target appeared in the upper group and 1 point for correct identification if the target appeared in the lower group; point assignments were reversed in the counterclockwise condition.

The above point-incentive system would no doubt encourage the desired scanning strategies, were such scanning possible. From the perspective of an independent-parallel-channels conception, however, the incentive could have had a significant nonperceptual role that would result in the data obtained. Such a conception (Eriksen \& Spencer, 1969; Gardner, 1970, 1973) postulates separate perceptual and decisional processing stages. Perceptual processing occurs in independent parallel channels subject to confusion errors (i.e., stimulus letters can be misidentified). The decisional stage assesses the imperfect information yielded by the perceptual stage and chooses the response for the trial. The Shaw and LaBerge point incentives would not influence perceptual processing-there being no spatial capacity limit or attentional control-but could influence the strategy of the decisional stage in its selection of a response. Given that correct identification of the target when it appeared in the low-value lacation was one-quarter as important as when it appeared in the high-value location, S could choose to lessen the weighting of perceptual information originating from the former location in arriving at a response decision. It can 
be demonstrated, furthermore, that such a strategy would result in poorer identification accuracy when the target appeared in the low-value location than when it appeared in the high-value location-exactly the pattern of results obtained by Shaw and LaBerge.

The effects of the strategy may be intuited as follows (see also Gardner, 1973): the $S$ in the Shaw and LaBerge experiment must make a single forced-choice response on the basis of imperfect information from two perceptual loci or channels-Channel A and Channel B; should the information from Channel $A$ veridically register the identity of an actual target, the response chosen can still be incorrect on trials when Channel B erroneously registers an incorrect target item (i.e., the decision maker can be misled by a confusion error on Channel B). If, however, the $S$ chooses to lessen the weight given to the information from Channel $B$ in arriving at a decision, he automatically increases his accuracy on trials when the target is presented to Channel A (the strategy tends to insulate the decision maker from confusion errors on B). ${ }^{1}$ Lowered weighting of information from Channel B, at the same time, decreases S's accuracy on trials when the target is presented to B (the strategy tends to insulate the decision maker from potentially veridical information about the target). The relative weighting placed on the two channels for optimal earnings in the experiment clearly depends on the incentive values employed and the degree to which nontarget letters tend to be confused with the target alternatives. Further, it may be assumed that the adoption of a given decisional strategy would require some practice and would be influenced by the particular spatial layout of the stimulus array as well as by biases from past learning.

The Shaw and LaBerge effect is thus easily interpreted within the framework of an independentparallel-channels conception of perceptual processing. The effect, furthermore, may be added to the list of results obtained in a variety of other detection experiments that are compatible with such a scheme (see Gardner, 1973). For this reason. the need for postulating either a perceptual capacity limit-serial (Shaw \& LaBerge) or parallel (Rumelhart, 1970)-or an attentional mechanism appears questionable.

\section{REFERENCES}

Eriksen, C. W., \& Spencer, T. Rate of information processing in visual perception: Some results and methodological considerations. Journal of Experimental Psychology Monograph, 1969, 79 (No. 2, Part 2).

Estes, W. K., \& Taylor, H. A. Visual detection in relation to display size and redundancy of critical elements. Perception \& Psychophysics, 1966, 1, 9-16.

Gardner, G. T. Spatial processing characteristics in the perception of brief visual arrays. (Doctoral dissertation.) Technical Report No. 23, Human Performance Center, University of Michigan, 1970.

Gardner, G. T. Evidence for independent parallel channels in tachistoscopic perception. Cognitive Psychology, 1973, 4, 130-155.

Rumelhart. D. E. A multicomponent theory of the perception of briefly exposed visual arrays. Journal of Mathematical Psychology, 1970, 7, 191-218.

Shaw, P.. \& LaBerge, D. Manipulation of visual processing by varying the rewards associated with display locations. Perception \& Psychophysics, 1971, 10, 348-352.

Shiffrin, R. M., \& Gardner. G. T. Visual processing capacity and attentional control. Journal of Experimental Psychology, $1972,93,72-82$.

\section{NOTE}

1. Within the framework of the ICC model developed by Gardner (1973), "lessening the weight" would, for example, correspond to dividing the appropriate perceptual sample by a constant factor before deciding on a response.

(Received for publication January 23, 1973; accepted February 7, 1973.) 